\title{
To clamp or not to clamp?
}

\section{Early venous and arterial vascular control improves blood loss in open radical prostatectomy}

\author{
Roderick Clark, Stacy Fan, Roshan Navaratnam, Nahid Punjani, Nicholas Power
}

\section{ABSTRACT}

Introduction: Radical prostatectomy (RP) is the goldstandard surgical treatment for men with clinically localized prostate cancer (PCa). Surgical techniques to minimize intra and post-operative complications are well established, but excessive bleeding during RP continues to be a concern. The objective of our study was to determine whether additional intraoperative temporary occlusion of the internal iliac arteries combined with a penile base tourniquet during open RP improves hemostasis.

Methods: We conducted a retrospective chart review of 23 patients who underwent open RP between Jan 2014 to May 2016. Eight patients underwent open RP with additional clamping of the internal iliac arteries using bulldog vascular clamps combined with a penile base Penrose drain tourniquet as temporary prostatic arterial and venous control during dorsal venous complex ligation and neurovascular bundle sparing. Our primary outcome was immediate and post-operative day 1 hemoglobin levels. Our main outcome was analyzed using Students t-test with equal variance.

Results: We stratified participants by clamping type. Fifteen patients underwent no clamping and 8 patients had the combined temporary clamping. Primary analysis of estimated blood loss showed a reduction in average blood loss among individuals with vascular control technique versus usual technique $(516 \mathrm{ml}$ and $754 \mathrm{ml}$ respectively, $\mathrm{p}=0.021)$. There were no obvious intraoperative or postoperative complications noted that could have been attributable to the temporary vascular control techniques.

Conclusion: Temporary vascular control with the addition of minor surgical techniques during open RP may improve an objective measure of blood loss immediately after surgery.

\section{INTRODUCTION}

Open radical prostatectomy (RP) is the gold-standard surgical treatment for men with clinically localized prostate cancer. The surgical technique for open nerve-sparing radical prostatectomy is well-established, but excessive bleeding continues to be a concern. ${ }^{1}$ This is an especially daunting task for a junior surgeon in the modern era interested in offering appropriate nerve-sparing and adequate lymph node dissection with satisfactory functional and oncologic outcomes. The learning curve has become potentially more difficult, given that surgical management of low-risk disease is almost non-existent in contemporary prostate cancer patients. ${ }^{2}$

Vascular control during open RP continues to be a challenge given the significant anatomical variability in prostatic arterial and venous systems. Improved hemostasis via clamping of the hypogastric arteries was first proposed by Peters et al and resulted in a significant reduction in the need for blood transfusions. ${ }^{3}$ This technique was evaluated in a randomized control trial with 130 patients, but did not have any major effect on blood loss parameters (pre- vs post-operative hematocrit). ${ }^{4}$ The authors attributed this finding to the extensive collateral vascular supply of the prostate and substantial contribution of uncontrolled venous blood loss. We aspired to develop a technique to improve venous control as a mechanism to reduce blood loss during open RP.

The objective of our study was to determine whether temporary intraoperative clamping of the internal iliac arteries combined with use of a Penrose drain as a tourniquet at the base of the penis during open RP improves perioperative blood loss for a junior surgeon still in his surgical learning curve. ${ }^{5}$

\section{METHODS}

We conducted a retrospective chart review of 29 patients who underwent radical prostatectomy between January 2014 to June 2016 by a single uro-oncologist within the first 5 years of practice at a tertiary care centre. Patients in our cohort were either given treatment as usual, or had additional clamping for early vascular control, based on the anticipated difficulty of the operation and in anticipation of increased blood loss.Patients in the vascular control group underwent clamping of the internal iliac arteries using bulldog vascular clamps in combination with a Penrose drain penile base tourniquet to provide temporary prostatic arterial and venous control during dorsal venous complex ligation and nerve sparing.

Our primary outcome of interest was peri-operative blood loss, derived from the post-operative note and confirmed quantitatively from post-operative bloodwork using the Bourke formula for estimating surgical blood loss. ${ }^{6}$ We used a simplified version of the Bourke formula (Figure 1). Data on patient demographics, perioperative and post-operative outcomes were collected. Student's t-test and chi-squared statistics were generated using Stata version 11.2. Ethics approval for this study was through the Western University Institutional Ethics Review Board. Informed consent was waived as the study is retrospective. All patient level data is confidential.

\section{RESULTS}

We identified 29 individuals who underwent open radical prostatectomy during the study period, of whom 15 belonged to the vascular control group. The average age was 63.5 years with a mean BMI of 28.2. Approximately one-third of patients (27.6\%) had a family history of prostate cancer and $27.6 \%$ were smokers 


\begin{tabular}{|c|}
\hline $\mathrm{EBL}=\mathrm{BV}^{*}\left(\mathrm{Hct}_{0}-\mathrm{Hct}_{4}\right)^{*}\left(3-\mathrm{Hct}_{\text {mean }}\right)$ \\
\hline EBL: Estimated blood loss \\
\hline BV: Estimated blood volume (Weight $(\mathrm{kg}) * 75)$ \\
\hline Hcto: Baseline hematocrit \\
\hline Hct: Postoperative hematocrit \\
\hline Hctmean: Mean hematocrit \\
\hline
\end{tabular}

Figure 1. Bourke formula to estimate perioperative blood loss

at the time of operation. Prostate biopsy showed mostly Gleason 7 prostate cancer (93.1\%) with significantly more individuals in the "surgery as usual group" with clinical T2a disease ( $\mathrm{n}=4$ vs $0 ., \mathrm{p}=$ 0.026 ) and most having clinical T2c disease in the vascular control group ( $\mathrm{n}=12$ vs $4 ., \mathrm{p}=0.005$ ) (Table 1 ).

Primary analysis of estimated blood loss showed a reduction in average blood loss among individuals with vascular control techniques versus the usual technique $(516 \mathrm{ml}$ and $754 \mathrm{ml}$ respectively, $\mathrm{p}=0.021)$. These findings were confirmed using the Bourke formula (1268 $\mathrm{ml}$ and $1956 \mathrm{ml}$ respectively , $\mathrm{p}=0.014)$. Final pathology showed that the majority of patients had T2c disease (44.8\%), with only 3 patients (10.3\%) having node positive disease postoperatively. There were no other differences observed between these two groups in terms of operative time, nerve sparing, duration of hospital stay, margin status, or rates of blood transfusion (Table 1).

\section{DISCUSSION}

We demonstrated that clamping of the internal iliac arteries using bulldog vascular clamps in combination with a Penrose drain tourniquet at the penile base during open radical prostatectomy was associated with a reduction in perioperative blood loss. While this study is not designed to demonstrate a causative relationship, we feel these results warrant further investigation. The cohort was unique as it was collected from a junior urologic oncologist in the first five years of practice. Although the duration of hospital stay and transfusion rates were similar between groups, given the small number of overall participants, it is possible that these differences will manifest in a larger trial. We postulate that the mechanism of

Table 1. Demographic, pathology, peri- and post-operative characteristics of study group

\begin{tabular}{|c|c|c|c|}
\hline & Standard RP $(n=14)$ & Early vascular control $(n=15)$ & p-value \\
\hline \multicolumn{4}{|l|}{ Demographic characteristics } \\
\hline Age (mean, SD) & $63.7(5.17)$ & $63.4(6.93)$ & 0.433 \\
\hline $\mathrm{BMI}$ (mean, SD) & $27.9(3.05)$ & $28.5(6.10)$ & 0.6355 \\
\hline Positive family history of prostate cancer & $5(35.7 \%)$ & $3(20.0 \%)$ & 0.344 \\
\hline Current smokers & $3(21.4 \%)$ & $5(33.3 \%)$ & 0.365 \\
\hline \multicolumn{4}{|l|}{ Pathology characteristics } \\
\hline \multicolumn{4}{|l|}{ TRUS Biopsy Gleason Score } \\
\hline 7 & $13(92.8 \%)$ & $14(93.3 \%)$ & 0.501 \\
\hline 8 & $1(7.1 \%)$ & $1(6.67 \%)$ & 0.960 \\
\hline \multicolumn{4}{|l|}{ Clinical T-stage } \\
\hline $\mathrm{T} 2 \mathrm{a}$ & $4(28.5 \%)$ & $0(0 \%)$ & 0.026 \\
\hline $\mathrm{T} 2 \mathrm{~b}$ & $6(42.8 \%)$ & $3(20 \%)$ & 0.184 \\
\hline $\mathrm{T} 2 \mathrm{C}$ & $4(28.5 \%)$ & $12(80 \%)$ & 0.005 \\
\hline \multicolumn{4}{|l|}{ Perioperative characteristics } \\
\hline Nerve sparing operation & $6(42.8 \%)$ & $6(40.0 \%)$ & 0.876 \\
\hline Estimated blood loss (ml) & $754.16(347.36)$ & $516.41(45.78)$ & 0.021 \\
\hline Bourke estimated blood loss $(\mathrm{ml})$ & $1956(184)$ & $1268(231)$ & 0.014 \\
\hline Operative time (minutes) & $188(8.76)$ & $192(7.92)$ & 0.652 \\
\hline \multicolumn{4}{|l|}{ Postoperative characteristics } \\
\hline Hospital stay (days) & $2.64(0.16)$ & $2.86(0.23)$ & 0.773 \\
\hline Blood transfusion & $1(7.14 \%)$ & $1(6.67 \%)$ & 0.960 \\
\hline \multicolumn{4}{|l|}{ Final Pathology } \\
\hline \multicolumn{4}{|l|}{ T stage } \\
\hline pT2c & $7(50.0 \%)$ & $6(40.0 \%)$ & 0.580 \\
\hline pT3a & $6(42.8 \%)$ & $5(33.3 \%)$ & 0.590 \\
\hline pT3b & $1(7.1 \%)$ & $4(26.6 \%)$ & 0.164 \\
\hline \multicolumn{4}{|l|}{ N stage } \\
\hline NO & $14(100 \%)$ & $12(80 \%)$ & -- \\
\hline N1 & $O(0 \%)$ & $3(20 \%)$ & 0.077 \\
\hline Margins positive & $4(28.5 \%)$ & $8(53.3 \%)$ & 0.176 \\
\hline Lymph nodes collected (median, IQR) & $5.5(3-10)$ & $8(5-11)$ & 0.236 \\
\hline
\end{tabular}


action of our intervention is a result of reduced venous backflow blood loss during dissection of the dorsal venous complex (DVC).

The single largest improvement in the technique of open RP regarding blood loss is undoubtedly the control of the DVC and prostatic pedicle introduced two decades ago. Since this innovation, several surgical and technical maneuvers have been attempted to reduce blood loss during open $\mathrm{RP}$, including patient positioning in Trendelenburg position with flexion of the hips, and the use of a Ligasure $^{\circledR}$ device or endoscopic gastrointestinal anastomosis stapler for hemostasis of the DVC. ${ }^{7-9}$ While several of these techniques are effective, some are associated with significantly increased perioperative cost. Our technique is simple and effective, and does not significantly extend operative time.

There are a few important limitations of our study. Firstly, it is a small, non-randomized, retrospective case series of a single surgeon, which carries a significant risk of bias. Secondly, surgeon estimates of total blood loss are unreliable. We attempted to mitigate this error by including a second objective measure of blood loss. Thirdly, we performed this study in a junior surgeon who is still on the learning curve for open prostatectomy. There is a paucity of data on the effects of surgeon seniority as it relates to outcomes in radical prostatectomy but we hope to examine this during our follow-up study. Finally, our study lacks long term follow-up of patient-focused outcomes including oncologic control, erectile function and continence.

\section{CONCLUSION}

Early vascular control of the internal iliac arteries with bulldog clamps and placement of a Penrose drain tourniquet at the penile base during open radical prostatectomy significantly reduces objective perioperative blood loss and does not significantly extend operative time. It is associated with almost no risk or increased cost. This technique can be adequately used for junior surgeons still within their learning curve to avoid major blood loss and still perform nerve-sparing extended lymph node dissection, and obtain adequate oncologic outcomes safely and effectively. Future research should focus on improving methodological quality of the study design to effectively evaluate these results. There may also be a role for this technique during open radical cystectomy in males.

\section{REFERENCES}

1. Yaxley JW, Coughlin GD, Chambers SK, et al. Robot-assisted laparoscopic prostatectomy versus open radical retropubic prostatectomy: early outcomes from a randomised controlled phase 3 study. Lancet. 2016 Sept;388(10049):1057-66. https://doi.org/10.1016/ $\underline{\text { S0140-6736(16)30592-X }}$

2. Silberstein JL, Vickers AJ, Power NE, et al. Reverse stage shift at a tertiary care center: escalating risk in men undergoing radical prostatectomy. Cancer. 2011 Nov;117(21):4855-60. https://doi. org/10.1002/cncr.26132

3. Peters CA, Walsh PC. Blood transfusion and anesthetic practices in radical retropubic prostatectomy. J Urol. 1985 Jul;134(1):81-3. https:// doi.org/10.1016/s0022-5347(17)46991-6

4. Kavoussi LR, Myers JA, Catalona WJ. Effect of temporary occlusion of hypogastric arteries on blood loss during radical retropubic prostatectomy. J Urol. 1991 Aug;146(2):362-5. https://doi.org/10.1016/ s0022-5347(17)37794-7

5. Vickers AJ, Bianco FJ, Serio AM, et al. The surgical learning curve for prostate cancer control after radical prostatectomy. J Natl Cancer Inst. 2007 Aug;99(15):1171-7. https://doi.org/10.1093/jnci/djm060

6. Bourke DL, Smith TC. Estimating allowable hemodilution. Anesthesiology. 1974 Dec;41(6):609-12.

7. Barré C, Pocholle P, Chauveau P. Minimal blood loss in patients undergoing radical retropubic prostatectomy. World J.Surg. 2002 Mar;26:1094-8. https://doi.org/10.1007/s00268-002-6226-7

8. Sengupta S, Webb DR. Use of a computer-controlled bipolar diathermy system in radical prostatectomies and other open urological surgery. ANZ J Surg. 2001 Sept;71(9):538-40. https://doi.org/10.1046/j.14401622.2001.02186.X

9. Muto G, Bardari F, Bozzo R, et al. Radical retropubic prostatectomy using endoscopic gastrointestinal anastomosis staplers. Eur Urol. 2001 Jan; 39 suppl 2:2-5. https://doi.org/10.1159/000052549 